\title{
ADAM28: Another ambivalent protease in cancer
}

\author{
Céline Hubeau $^{\mathrm{a}}$, Natacha Rocks ${ }^{\mathrm{b}}$, Didier Cataldo ${ }^{\mathrm{a}, \mathrm{c}, *}$ \\ a Laboratory of Tumor and Development Biology, GIGA-Cancer, University of Liège, Liège, Belgium \\ ${ }^{\mathrm{b}}$ Laboratory of Pharmaceutical Technology and Biopharmacy, CIRM, University of Liège, Liège, Belgium \\ ${ }^{\mathrm{c}}$ Department of Respiratory Diseases, CHU of Liège, University of Liège, Liège, Belgium
}

\section{A R T I C L E I N F O}

\section{Keywords:}

ADAM28

Cancer biomarker

Cell proliferation

Metastasis

Tumor microenvironment

\begin{abstract}
A B S T R A C T
Emergence of novel therapeutic options in a perspective of personalized therapy of cancer relies on the discovery of precise molecular mechanisms involved in the switch from a localized tumor to invasive metastasis spread. Pro-tumor functions have been mostly ascribed to proteolytic enzymes from the metalloproteinase family including A Disintegrin And Metalloproteinases (ADAMs). Particularly, when expressed by cancer cells, ADAM28 protease supports cancer cell proliferation, survival and migration as well as metastatic progression. In sharp contrast, ADAM28 derived from the tumor microenvironment has shown to exert strong protective effects against deleterious metastasis dissemination. Indeed, depletion of host-derived ADAM28 (ADAM28 KO mice) accelerates colonization lung tissues, increases tumor foci implantation, and impairs $\mathrm{T}$ cell immune response.

In this review, we outline specific ADAM28 functions when specifically expressed by carcinoma cells or by tumor microenvironment. Finally, we discuss about future research strategies that could be pursued to highlight new functions of this protease in cancer.
\end{abstract}

\section{Introduction}

Members of ADAM (A Disintegrin And Metalloproteinase) family belong to the superfamily of zinc-dependent metalloproteinases, known as metzincins, which also comprises MMPs (Matrix Metalloproteinases) and ADAMTS (A Disintegrin And Metalloproteinase with ThromboSpondin motifs). Metzincin proteases are characterized by a highly conserved catalytic domain containing the zinc-binding consensus sequence required for proteolytic activity. Together with ADAMTS, ADAMs constitute the subfamily of adamalysins which can be distinguished from MMPs by the presence of a disintegrin domain. Discovery of ADAMs has emerged with the identification of a surface protein called PH-30 (ADAM1) that plays a role in sperm-egg fusion during fertilization [1]. Twenty-two ADAMs have been reported in human while 32 ADAMs have been identified in mouse [2]. ADAM proteases are large membrane-anchored glycoproteins composed of different domains exerting multiple functions including proteolysis, adhesion, cell fusion and intracellular signaling. Although ADAMs contribute to diverse physiological functions, they are also associated with pathological conditions such as cancer. Indeed, implication of ADAMs in cancer has been extensively described in the literature since they can modulate various processes including proliferation, apoptosis, migration and invasion of tumor cells as well as angiogenesis [3-5]. However, accumulating evidence demonstrates that metalloproteinases such as ADAMs can also exert protective effects against tumor progression [6].

\section{ADAM28 features}

In 1999, by cloning the human lymph node cDNA library, Roberts and colleagues have identified a novel protein, bearing metalloprotease and disintegrin domains, that has been firstly named MDC-L (for Metalloprotease Disintegrin Cysteine-rich-Lymphocyte) [7]. Later, since it is also expressed by other cells and because its structure is highly conserved and similar to ADAM proteases, MDC-L has been assigned to the ADAM family and re-named ADAM28. The human ADAM28 gene is located on chromosome 8 p24.2 whereas the mouse ADAM28 gene is found on chromosome 14. Due to an alternative splicing, the ADAM28 protein exists as two different isoforms: a classical transmembrane form (ADAM28m) and a shorter (secreted) form, lacking the transmembrane domain (ADAM28s) $[8,9]$. Both isoforms, ADAM28m and ADAM28s, are initially produced as proforms of 87 and $65 \mathrm{kDa}$, respectively, which can be further processed to generate proteolytically active forms of 55/57 and $42 \mathrm{kDa}$, respectively. Membrane-bound ADAM28 depicts the common structure of an ADAM protease and is composed from $\mathrm{N}$ - to

\footnotetext{
* Corresponding author. University of Liège, Tower of Pathology (B23), 3rd floor, 4000, Liège, Belgium.

E-mail address: didier.cataldo@uliege.be (D. Cataldo).
} 
C-terminus of the following domains: a signal peptide, a pro-domain, a metalloproteinase domain, a disintegrin, a cysteine-rich, an EGF-like, a transmembrane and a cytoplasmic domain (Fig. 1). Structure of secreted ADAM28 is similar to transmembrane ADAM28 except that the cysteine-rich domain is shorter whereas EGF-like, transmembrane and cytoplasmic domains are missing (Fig. 1). While the signal peptide allows to correctly address proteases towards the secretory pathway, the pro-domain sequesters the metalloproteinase domain through a cysteine-switch mechanism characterized by the binding of a conserved cysteine residue to the zinc cation in the catalytic site and thereby, maintains the protease into an inactive state. Consequently, protease activation requires subsequent cleavage of the pro-domain. Unlike most of other ADAM proteases that require furin-convertases for pro-domain removal, activation of ADAM28 can be either autocatalytic, either mediated by the matrix metalloprotease MMP-7 [8-10]. Indeed, recombinant inactive ADAM28 is auto-activated upon storage in solution at $4{ }^{\circ} \mathrm{C}$ suggesting an autocatalytic activation mechanism for this protease [10]. ADAM28 is also processed by MMP-7 leading to the release of active ADAM28 lacking the pro-domain [9]. ADAM28 possesses the HEXGH consensus sequence within its metalloproteinase domain allowing the binding of zinc and therefore, exhibits catalytic activity. Proteolytic activity of ADAM28 has been firstly demonstrated in vitro through the cleavage of recombinant MBP (myelin basic protein) [11]. Other substrates have been further identified upon several studies such as CTGF (Connective Tissue Growth Factor) [12], IGFBP-3 (Insulin-like
Growth Factor-Binding Protein-3) [9,13], vWF (von Willebrand Factor) [14] and Notch2 [15] (Table 1). ADAM28 could modulate angiogenesis since CTGF is a potent angiogenic inducer which has been associated with tumorigenesis and angiogenesis in vivo [12,16-18]. Moreover, this growth factor can exert antiangiogenic effect by interacting with VEGF-A (Vascular Endothelial Growth Factor-A) to prevent binding to

Table 1

Summary of ADAM28 substrates.

\begin{tabular}{|c|c|c|c|}
\hline Name & $\begin{array}{l}\text { Biological } \\
\text { significance }\end{array}$ & Common ADAM & References \\
\hline MBP & Myelination process & ADAM10 & Howard et al., 2001 \\
\hline $\mathrm{CD} 23$ & Allergic response & $\begin{array}{l}\text { ADAM- }-8,-10 \text { and } \\
-15\end{array}$ & Fourie et al., 2003 \\
\hline $\begin{array}{l}\text { IGFBP- } \\
3\end{array}$ & Proliferation & ADAM12 & $\begin{array}{l}\text { Mochizuki et al., } \\
2004\end{array}$ \\
\hline CTGF & Angiogenesis & ADAMTS7 & $\begin{array}{l}\text { Mochizuki et al., } \\
2010\end{array}$ \\
\hline TNF- $\alpha$ & Inflammation & ADAM17 & Jowett et al., 2012 \\
\hline CD200 & Immunoregulation & & Tiwto et al., 2013 \\
\hline vWF & Hemostasis & ADAMTS13 & $\begin{array}{l}\text { Mochizuki et al., } \\
2013\end{array}$ \\
\hline Notch2 & B cell maturation & ADAM10 & Zhang et al., 2017 \\
\hline
\end{tabular}

Abbreviations: MBP, Myelin Basic Protein; IGFBP-3, Insulin-like Growth Factor Binding Protein-3; CTGF, Connective Tissue Growth Factor; TNF- $\alpha$, Tumor Necrosis Factor-alpha; vWF, von Willebrand Factor.

\section{ADAM28m}

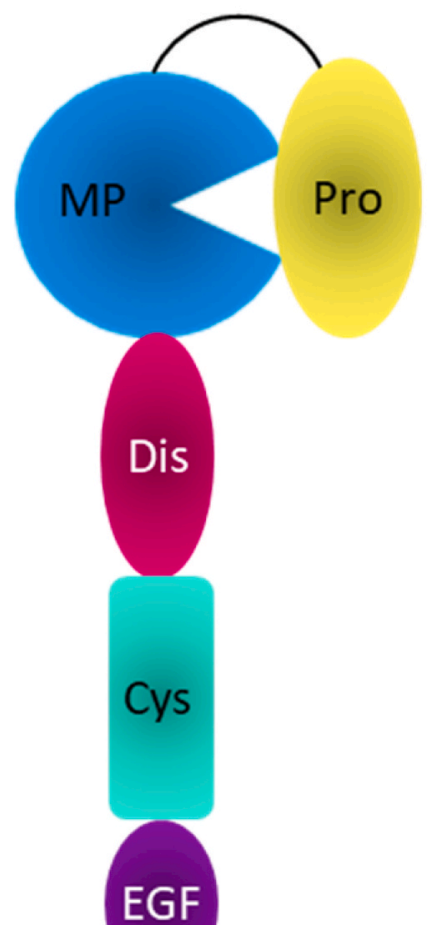

ADAM28s

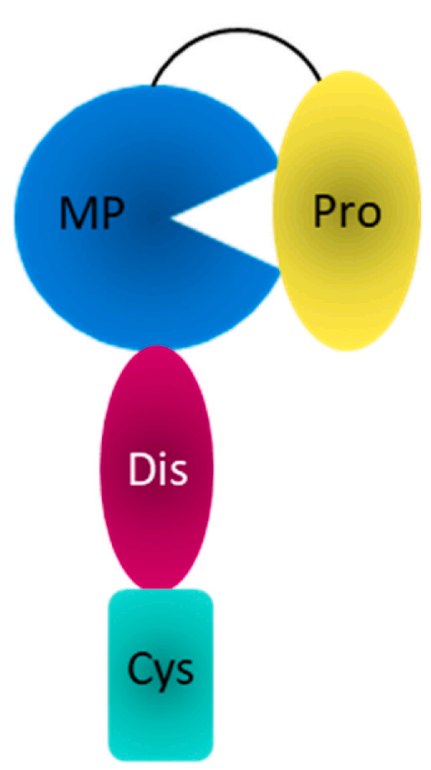

Fig. 1. Structure of ADAM28 isoforms. Transmembrane ADAM28 (ADAM28m) is composed of different functional domains: prodomain (Pro), metalloproteinase domain (MP), disintegrin domain (Dis), cysteine-rich domain (Cys), EGF-like domain (EGF), a transmembrane domain (TM) and a cytoplasmic tail (Cyt). Secreted form of ADAM28 (ADAM28s) shares the similar structure except that the EGF-like, transmembrane and cytoplasmic portions are missing. 
its receptor (VEGFR) and thus, attenuate VEGF signaling pathway [19]. IGFBP-3 is the main carrying protein that transports IGFs (Insulin-like Growth Factors) in the blood circulation and regulate their bioavailability for tissues. Through IGFP-3 cleavage, ADAM28 could cause IGFs release and thereby, stimulate cell proliferation [9]. Besides, ADAM28 could also influence hemostasis and angiogenesis in physiological conditions via vWF processing. Indeed, vWF exhibits pro-apoptotic properties by directly inducing tumor cell death and thereby, reducing metastasis formation suggesting an involvement of ADAM28 in cell survival [14]. Moreover, a recent study has highlighted that ADAM28 could be involved in Notch 2 processing and therefore, could play a role in various development mechanisms since Notch2 controls cell fate decisions [15]. Protein sequence analysis indicates that ADAM28 and SVMPs (Snake Venom MetalloProteinases) structures are closely related indicating that they could cleave common substrates such as components of the extracellular matrix (type IV collagen, laminin, fibronectin, gelatins and proteoglycans) suggesting a potential role of ADAM28 in tissue remodeling $[8,20]$.

In addition to their sheddase activity, ADAM proteases mediate cellcell and cell-matrix interactions through the binding of their disintegrin domain to various molecules including integrins (Table 2). In particular, ADAM28 binds to $\alpha_{4} \beta_{1}, \alpha_{4} \beta_{7}$ and $\alpha_{9} \beta_{1}$ integrins and promotes adhesion of lymphocytes to leukocytes and endothelial cells as well as their migration [21-24]. By interacting with PSGL-1 on leukocytes, ADAM28 enhances PSGL-1/P-selectin mediated leukocyte rolling adhesion to endothelial cells and further, infiltration of these cells into tissues [25]. Therefore, ADAM28 could facilitate leukocyte infiltration under acute inflammatory conditions by modulating the initial step of the transendothelial migration of leucocytes. Besides, ADAM28 interferes with $\mathrm{C} 1 \mathrm{q} / \mathrm{C} 1 \mathrm{qR}$ interaction through binding to $\mathrm{C} 1 \mathrm{q}$ resulting in an attenuated cytotoxic response indicating that ADAM28 can protect bronchial epithelium against C1q-induced apoptosis [26]. The C-terminal extremity of ADAM28 encompasses a transmembrane domain allowing a solid anchorage to cell membrane and is followed by a cytoplasmic tail. Intracellular portion of ADAMs usually contains proline-rich regions that serve as ligands for SH3 (Src Homology 3) domain-containing proteins $[27,28]$.

Catalytic activity of ADAM proteases is regulated by Tissue Inhibitors of Metalloproteinases (TIMPs). ADAM28 proteolytic activity is found completely inhibited by TIMP-3 and TIMP-4 while TIMP-1 and TIMP-2 had no effect $[9,11]$. In addition, KB-R7785, a synthetic compound containing a zinc-chelating hydroxamic acid, has been shown to abolish ADAM28 proteolytic activity at a concentration of $1 \mu \mathrm{M}$ and can be used as an effective ADAM28 inhibitor in vitro [9,13,29]. However, it is important to note that the KB-R7785 inhibitor has off-target effects on diverse MMPs as well as ADAM12 and therefore, should not be considered as a specific inhibitor of ADAM28 [30].

Table 2

Summary of ADAM28 protein partners.

\begin{tabular}{clll}
\hline Name & Biological significance & Common ADAM & References \\
\hline Integrin & Cell adhesion & ADAM-2, $-3,-7$, & Bridges et al., \\
$\alpha_{4} \beta_{1}$ & & -12 and -33 & 2001 \\
Integrin & Cell adhesion & ADAM7 & Bridges et al., \\
$\alpha_{4} \beta_{7}$ & & & 2005 \\
Integrin & Cell adhesion & ADAM-7 and -12 & Bridges et al., \\
$\alpha_{9} \beta_{1}$ & & & 2005 \\
PSGL-1 & Transendothelial migration & & Shimoda et al., \\
& & & 2007 \\
C1q & Innate immune response via & & Miyamae et al., \\
& complement system & 2016 \\
\hline
\end{tabular}

Abbreviations: PSGL-1, P-Selectin Glycoprotein Ligand-1; C1q, Complement component 1q.

\section{Physiological expression}

ADAM28 mRNA expression has been primarily identified in human lymphocytes extracted from lymph nodes but its expression is also observed in lymphocytes from blood and other secondary lymphoid tissues such as the spleen [7]. ADAM28 protein is further found on the surface of peripheral blood lymphocytes as well as in T- and Epstein-Barr virus-transformed B-lymphocyte cell lines suggesting a function of this protease in the immune system [7]. Using more recent microarray and real-time PCR assays or a dataset (GeneAtlas U133A), McGinn's group has extended previous findings on ADAM28 expression and has reported high ADAM28 expression in the spleen and in peripheral blood leucocytes [23] as well as in $\mathrm{CD} 19^{+} \mathrm{B}$ cells (www.biogps.org). Based on these expression profiles and since CD19 is a major co-receptor of the B cell Receptor (BCR) that is crucial for B cell development, one could hypothesize a possible implication of ADAM28 in B cell maturation and differentiation as supported by a study indicating that ADAM28 could participate in the differentiation of marginal zone precursors to marginal zone B cells in the spleen [15]. Further studies have demonstrated ADAM28 expression in epithelial cells of various organs including epididymis, bronchus and stomach [26].

While the homology of ADAM28 protein is about 70\% between human and mouse species, the ADAM28 expression pattern in these two species is quite different. High levels of ADAM28 are found in the epididymis of mice $[8,31]$. ADAM28 is also expressed by thymic epithelial cells in fetus and adult mice indicating that this protease could regulate $\mathrm{T}$ lymphocyte differentiation as it has already been reported for ADAM8, ADAM10 and ADAM17 [32-38]. ADAM28 has also been identified in the respiratory tract of mice (lung, trachea) [32]. Unlike human tissues, no detectable ADAM28 mRNA has been observed in the murine spleen nor on isolated $\mathrm{CD}^{+} \mathrm{T}$ lymphocytes [8,33]. However, gene and immunochemistry analyses reveal that murine ADAM28 is predominantly and specifically expressed by B cells from the marginal zone of the spleen $[23,39]$. Further flow cytometry data confirm that ADAM28 is highly expressed by B-cell marginal zone precursors suggesting a potential function of ADAM28 in murine B cell development [15]. At the present time, no clear and distinct expression pattern has been highlighted between both transmembrane and secreted ADAM28 in human and murine tissues. As described below, no available data suggest specific functions of each isoform in physiological and pathological processes including cancer.

\section{Implication of ADAM28 in physiological and pathological processes}

Initial investigations on ADAMs have indicated that these proteases could play a major role in fertilization process as first discovered ADAMs (ADAM1 and ADAM2) are involved in sperm-egg plasma membrane fusion [1,40-42]. Later, additional ADAMs including ADAM28 have been found specifically expressed in the male reproductive tract confirming a potential function in fertilization [42-44]. However, no fertility issues could be highlighted when the expression of specific ADAMs is turned off in viable murine knockout mouse models indicating that isolated ADAM proteases are not crucial for mouse reproduction. Specific depletion of ADAM28 in mice neither affects spermatogenesis nor female fertility demonstrating that ADAM28 is not essential for reproduction [45] (and unpublished data).

As aforementioned, ADAM28 expression has been firstly identified in lymphocytes suggesting a predominant function in the immune system. Hence, ADAM28 expression by thymic epithelial cells could indicate a key role in T lymphocyte differentiation [32]. Previous studies have revealed that mice depleted for ADAM8, ADAM10 or ADAM17 displayed abnormal thymocyte development and maturation, partial inhibition of early $\mathrm{T}$ cell development and maturation or impaired intrathymic migration of single positive $\mathrm{T}$ cells to the medulla [34-38]. In contrast, mice deficient for ADAM28 show a normal spleen morphology 
associated to normal thymocyte maturation implying that the presence of ADAM28 is not essential for a smooth functioning of splenic activities [33]. ADAM28 could also be implicated in B cell maturation, a multi-step process characterized by the emigration of immature B cells from the bone marrow to the spleen where they will undergo differentiation steps to become either marginal zone (MZ) or follicular (FO) B cells. For example, ADAM10 has been reported to regulate MZ B-cell development by initiating Notch2 signaling as demonstrated by an altered B cell development and impaired Notch2 signaling observed in B cell-specific ADAM10 knockout mice [37,39,46]. A CD19-deficient mouse model highlights the relevance of ADAM28 in B cell maturation [15]. Indeed, the B cell surface co-receptor CD19 is critical to ensure the appropriate differentiation of marginal zone precursor (MZP) B cells into MZ B cells [47]. In mice, CD19 depletion completely abolishes this process and is associated with a reduced expression of ADAM28 in MZP cells. Conversely, ADAM28 expression levels can be restored in these cells by inducing CD19 expression indicating that CD19 regulates ADAM28. Additional results demonstrate that CD19, via the inhibition of the transcription factor Foxo 1, concomitantly upregulates ADAM28 and Notch2-controlled genes suggesting that cleavage of Notch2 by ADAM28 might contribute to MZ B cells differentiation. However, further investigations are required to confirm Notch2 cleavage by ADAM28 and confirm this hypothesis.

\subsection{Potential role of ADAM28 in immunological disorders and inflammation}

ADAM28 could be involved in immunological disorders including allergy, asthma and leukemia. Indeed, ADAM28 is a sheddase for the low affinity IgE receptor $\mathrm{CD} 23$, a transmembrane protein present on the surface of B cells, monocytes, macrophages and eosinophils [10,48]. Cleavage of $\mathrm{CD} 23$ proteins generates soluble forms (sCD23) that increase IgE release by B cells and induce production of pro-inflammatory mediators by macrophages [48]. Elevated levels of both membrane-bound and soluble CD23 are associated with pathological conditions like asthma, rheumatoid arthritis and inflammatory bowel disease. In chronic lymphocytic leukemia (CLL), ADAM28 expression is correlated with soluble CD200 (sCD200) levels in the plasma of patients [49]. The glycoprotein CD200 is expressed by various cell types including B lymphocytes and by interacting with its receptor expressed on cells from myeloid and lymphoid lineage, delivers immunomodulatory signals to suppress T cell-mediated anti-tumoral responses [50]. CD200 expression is upregulated in various malignancies including acute myeloid leukemia (AML) and chronic lymphocytic leukemia (CLL) and is associated with a poor prognosis in terms of overall survival of patients with AML [50]. Downregulation of ADAM28 in B cells from CLL patients using siRNA reduces the release of sCD200 suggesting that ADAM28 induces shedding of CD200, recognized as a prognostic marker [49].

In atherosclerosis, ADAM proteases exert multiple functions including cytokine regulation, cell adhesion and migration and therefore, could influence the behavior of infiltrating-monocytes at the site of atherosclerosis plaques. A gene analysis has been performed on THP-1 monocytes stimulated either with LDL obtained from type-2 diabetes patients or with LDL isolated from control subjects and results indicate that ADAM28 expression by monocytes is induced upon stimulation of THP-1 cells with LDL derived from type-2 diabetes patients [51]. Moreover, high levels of ADAM28 have been measured on blood mononuclear cells of patients with metabolic syndrome [52]. Besides, ADAM28 is downregulated in monocytic THP-1 cells after the treatment with Imatinib, a selective tyrosine kinase inhibitor that could be atheroprotective by decreasing the monocytic cholesterol uptake [53]. Altogether, these data suggest that ADAM28 could be involved in atherosclerosis by regulating monocyte adhesion to endothelium and subsequent migration into subendothelial tissues.

Upregulation of ADAM28 expression is observed in high fat diet-fed mice at both RNA and protein levels [54]. To determine whether ADAM28 can influence parameters linked to the metabolic syndrome, mice have been treated with a high fat diet as well as with ADAM28-directed siRNA, which reduces ADAM28 expression by at least $50 \%$. ADAM28 downregulation is associated with reduced weight gain, reduced seric urea and liver enzyme levels as well as a decrease of TNF- $\alpha$ within the liver [54]. Notably, ADAM28 is a potential sheddase of TNF- $\alpha$ since co-expression of ADAM28 and TNF- $\alpha$ in HEK-293 cells is associated with the release of mature TNF- $\alpha$ in the cell-free supernatant [52]. Hence, a reduced cleavage of TNF- $\alpha$ is observed after inhibition of endogenous ADAM28 in stimulated macrophages and monocytes. Plausible implication of ADAM28 in metabolic syndrome has been further confirmed by totally depleting ADAM28 in mice [54]. Several metabolic parameters have been analyzed in both ADAM28 KO and WT mice following a normal chow diet. Depletion of ADAM28 is significantly correlated with elevated levels of HDL considered as good cholesterol. In addition, kidney and liver functions improved in ADAM28 KO mice in comparison to WT mice.

Aside its proteolytic activity, ADAM28 modulates cell adhesion and migration of leucocytes through the interaction with three distinct integrins: $\alpha_{4} \beta_{1}$ [21-23], $\alpha_{4} \beta_{7}$ [24] and $\alpha_{9} \beta_{1}$ [24]. Disintegrin domain of ADAM28 binds to $\alpha_{4} \beta_{1}$ integrin (Very Late Antigen-4, VLA-4) expressed on lymphocyte membrane and might contribute to lymphocyte-leukocyte interaction [21,22,24]. Moreover, ADAM28 modulates lymphocytes $\alpha_{4} \beta_{1}$ binding to VCAM-1 (Vascular Cell Adhesion Molecule-1) expressed by endothelial cells that contributes to transendothelial migration [23]. Also, ADAM28 interacts with integrin $\alpha_{4} \beta_{7}$ (Lymphocyte Peyer patch Adhesion Molecule, LPAM) which ensures the adequate T-cell homing into gut-associated lymphoid tissues via MadCAM (Mucosal addressin Cell Adhesion Molecule) [24,55]. However, biological relevance of ADAM28/integrin $\alpha_{4} \beta_{7}$ interaction has not been determined. Moreover, ADAM28 interacts with $\alpha_{9} \beta_{1}$ integrin that mediates adhesion and transendothelial migration of neutrophils and inhibits their apoptosis [24,56]. However, it is not yet fully understood whether these interactions play a significant role in biological processes.

ADAM28 also promotes the PSGL-1/P-selectin-mediated leukocyte adhesion to endothelial cells [25]. Via its disintegrin domain, ADAM28 interacts with PSGL-1 and contributes to immune cells infiltration into inflamed tissues. Another ligand of ADAM28 is C1q, a complex glycoprotein involved in the innate immune response via the complement system and in the initiation of various cell responses by binding to its receptor [57]. Both, ADAM28 and C1q, are expressed on the surface of epithelial cells in normal bronchial tissues and in cultured bronchial epithelial cells [26]. It has been shown that ADAM28 attenuates C1q-mediated cell death whereas its downregulation accelerates this mechanism [26].

\subsection{Redundancy in the ADAM family and tools to study ADAM28}

Given that most ADAM28 substrates are shed by multiple ADAMs, the exact role of ADAM28 among other members of ADAM family in these different physiological and pathological processes might remain unclear (Table 1). Hence, determining precise functions of ADAM28 has challenging aspects since ADAM proteases could exert overlapping and compensatory effects as previously reported [35]. Therefore, knock-out mouse models provide a unique and precious tool to study roles of ADAM28. ADAM28 full knock-out mouse has been developed and introgressed into two mouse strains (C57BL/6JRj and Balb/cJrj) [33]. ADAM28 full knock-out (KO) mice are viable, fertile, do not display any apparent spontaneous phenotype or abnormal behavior. Interestingly, while ADAM28 is highly expressed in the epididymis and lymphocytes, no morphological alteration has been found in epididymis as well as a defect in $\mathrm{T}$ cell differentiation within the thymus after ADAM28 depletion [33] (and unpublished data). Although ADAM28 appears to be crucial for B cell maturation, no specific defect of this process could also 
be highlighted in the spleen of ADAM28 KO mice (unpublished data). Due to its potential functions in lipid metabolism, weight of both wild-type (WT) and ADAM28 KO mice have been monitored over time and no difference could be observed (unpublished data). However, we demonstrate that ADAM28 depletion is associated with increased dissemination of different cancer cells to lung tissues (see below). Another strain of ADAM28 KO mouse has been reported in the literature and its phenotype has been investigated exclusively on female mice. These ADAM28 KO mice tend to exhibit a reduced body weight at 10 months of age compared to WT mice. Moreover, elevated high-density lipoprotein cholesterol levels have been observed in the serum of ADAM28 KO mice suggesting that ADAM28 depletion alters metabolic pathways [54]. Nonetheless, this study has been performed on relatively small cohorts (3-4 mice/group) and the body weight and metabolic parameters measurements have been performed on mice with a different age. Therefore, more investigations are required to confirm this hypothesis such as the evolution of the body weight over time in both female and male mice and the evaluation of the different metabolic parameters in a larger group of mice at the same age. The other strain was tested regarding spontaneous cancer or metastasis.

\section{Why is ADAM28 an ambivalent protease in cancer?}

ADAM28 is overexpressed by carcinoma cells in multiple cancer types: breast cancer [9,12,13], renal cell carcinoma [58], non-small cell lung carcinoma (NSCLC) $[59,60]$, leukemia $[49,61]$, prostate cancer [29], chondrosarcoma [62], bladder cancer [63], pancreatic cancer [64] and head and neck carcinoma [65]. In experimental in vitro models, ADAM28 expression is induced by the protein kinase src in v-src-transformed epithelial cells and in various human carcinoma cells including PC-9 and MDA-MB231 cells [66]. Both PI3K/Akt and MEK/ERK signaling pathways are thought to be the downstream effectors of src and mediate ADAM28 expression. Likewise, PI3K/Akt signaling pathway has been shown to regulate ADAM28 expression in B-cell acute lymphoblastic leukemia [61]. In NSCLC, expression levels of ADAM28 are correlated with tumor size, lymph node metastasis and cell proliferation indicating an association between ADAM28 and cancer progression [59,60,67]. The impact of ADAM28 expression has been studied on disease-free survival after operation and overall survival of patients with NSCLC [60]. According to ADAM28 immunodetection in carcinoma tissues, three groups of patients have been determined as the following: a high-expressing, a medium-expressing and a low-expressing group. Results indicate that disease-free survival after operation is significantly lower in the high-expressing group compared to both medium-expressing and low-expressing groups. Overall survival of patients from the high-expressing group seems to be lower when compared to others groups although it is not statistically significant. In B-cell acute lymphoblastic leukemia (B-ALL) ADAM28 is a potential prognostic factor as its expression in bone marrow is associated with disease relapse which obviously compromises patient survival [61]. Indeed, ADAM28 expression is higher in patients experiencing disease relapse as compared to patients with favorable outcomes. Hence, increased ADAM28 expression correlates with lower probabilities of relapse-free survival (RFS) and event-free survival (EFS). Therefore, ADAM28 could be used as a biomarker to evaluate potential relapse in B-ALL patients. Moreover, ADAM28 has been reported as a valuable prognostic marker for acute myeloid leukemia. In this form of leukemia, high ADAM28 expression is associated with higher cumulative incidence of relapse as well as a worse overall survival and event-free survival [68]. Taken together, these data demonstrate that ADAM28 is a promising biomarker to predict relapse in patients with different types of leukemia. Since its expression is detectable in biological samples of patients (i.e. serum and urine), ADAM28 is also considered as an interesting biomarker for the diagnosis of several cancer types such as chondrosarcoma [62]. Indeed, ADAM28 upregulation correlates with the histologic grade of conventional chondrosarcoma and thus, ADAM28 could be useful to distinguish low-grade chondrosarcoma and enchondroma which are somewhat difficult to differentiate. Besides, ADAM28 expression is higher in tumors from patients displaying asbestos-related lung cancer than in tumors from patients with non-asbestos related lung cancer suggesting that ADAM28 is a potential oncogene in this cancer and could be a biomarker for asbestos-related lung cancer [69]. In pancreatic cancer, ADAM28 contributes to gemcitabine resistance and is a factor of poor prognosis in terms of overall survival (OS) and relapse-free survival (RFS) since patients with high ADAM28 expression exhibit a worse OS and RFS [64]. Many studies report the contribution of ADAM28 to development and progression of various cancers and some consider ADAM28 as a potential therapeutic target [3,4,70,71]. However, a recent study highlights a protective role of host-derived ADAM28 against tumor cell dissemination suggesting a dual role of this protease in cancer [33].

This duality of ADAM28 in cancer development will be discussed in the following paragraphs.

\section{Pro-tumorigenic effects of ADAM28}

\subsection{Cancer cell proliferation}

Cancer cell proliferation can be regulated by ADAM proteases through ectodomain shedding of growth factors and receptors. In vitro, ADAM28 is expressed by human breast carcinoma MDA-MB231 cell line and by various human lung cancer cell lines (PC-9, Calu-3, A549, Lu-1, H1650 and SK-MES) $[13,14,60]$. In patients with breast and non-small cell lung carcinoma, ADAM28 is upregulated at mRNA and protein levels in carcinoma tissues and ADAM28 mRNA expression is correlated with cell proliferation whereas no expression is observed in non-neoplastic tissues [13,59]. Especially, ADAM28 promotes cell proliferation via IGF-1 (Insulin-like Growth Factor-1) signaling axis. Indeed, downregulation of ADAM28 decreases cell proliferation of IGF-1-treated breast cancer cells in vitro [13]. Cleavage of IGFBP-3 by ADAM28 is indeed observed and enhances the bioavailability of IGF-1 [9]. Activation of IGFR initiates an intracellular signaling cascade via the phosphorylation of the downstream molecule ERK1/2 leading to an increased cell proliferation. Therefore, ADAM28 releases IGF-1 through the cleavage of IGFBP-3 and leads to activation of the IGF signaling pathway and finally, increases tumor cell proliferation (Fig. 2). In line, ADAM28 downregulation in vivo has been associated with a reduced tumor growth in a mouse model of mammary MDA-MB231 cell xenografts. A similar mechanism has been recently ascribed in acute myeloid leukemia (AML) where ADAM28 is described to be upregulated in the bone marrow of patients with AML, who could be assigned to a high or a low ADAM28 expression group, whereas healthy individuals do not show increased ADAM28 levels [68]. High ADAM28 expression is correlated with disease relapse as well as with AML dissemination in non-hematopoietic tissues and a higher proliferation capacity of primary blast cells isolated from AML patients. This is associated with increased degradation of IGFBP-3 leading to increased IGF-1-induced proliferation. Moreover, in vitro knock-down of ADAM28 in the acute myeloid leukemia HL-60 cell line reduces cell proliferation, migration and invasion [68]. A preliminary study has evaluated expression of ADAM28 and IGFBP-3 in patients with colorectal cancer using the microarray technique and transcriptomic analysis reveals that both ADAM28 and IGFBP-3 are differentially expressed when comparing transcriptomes of normal tissue with colorectal cancer from overweight/obese patients [72].

\subsection{Cancer-associated angiogenesis}

Angiogenesis is characterized by the spread of new blood vessels from pre-existing ones in response to pro-angiogenic factors. Members of the VEGF (Vascular Endothelial Growth Factor) family are the predominant factors stimulating the angiogenic process through binding to 


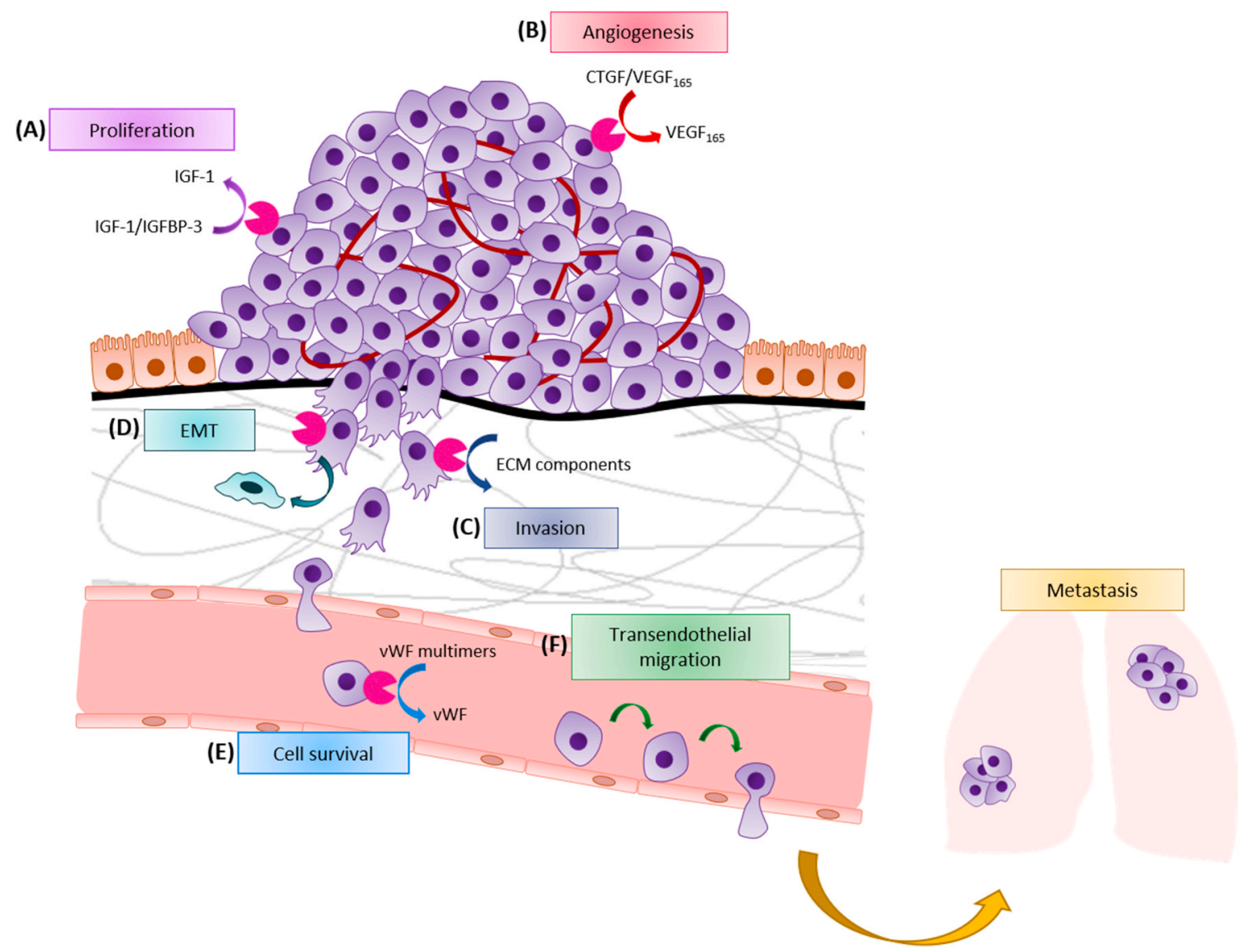

Fig. 2. Functional roles of ADAM28 in cancer progression. Upregulation of ADAM28 protease in diverse cancers is associated with tumor progression and metastatic dissemination. Identification of ADAM28 substrates allowed the characterization of pathways mediated by ADAM28 to promote cancer progression. (A) Through the cleavage of IGFBP-3, ADAM28 induces IGF-1 signaling axis to further stimulate cell proliferation. (B) Moreover, ADAM28 modulates the angiogenic process by releasing the proangiogenic factor VEGF via the dissociation of CTGF/VEGF complex. Expression of ADAM28 also contributes to invasiveness of cancer cells by different mechanisms. (C) Given its similarity with SVMPs, ADAM28 could degrade components of the ECM and thus, be involved in ECM remodeling. (D) Hence, ADAM28 gene expression mediates epithelial to mesenchymal transition process via the SOX4 transcription factor. (E) Proteolysis of vWF multimers in the blood circulation protects cancer cells from cell death. (F) Interaction between ADAM28 and adhesion molecules could facilitate the binding of cancer cells to endothelial cells, thereby enhancing transendothelial migration into surrounding tissues. Abbreviations: IGFBP-3, Insulin-like Growth Factor-Binding Protein-3; IGF-1, Insulinlike Growth Factor-1; VEGF, Vascular Endothelial Growth Factor; CTGF, Connective Tissue Growth Factor; SVMPs, Snake Venom Metalloproteinases; ECM, Extracellular Matrix; SOX4, SRY-related HMG-box 4; vWF, von Willebrand Factor.

VEGFRs (Vascular Endothelial Growth Factor Receptors). Notably, angiogenic activity of VEGF is regulated by CTGF (Connective Tissue Growth Factor), an extracellular matrix-associated protein belonging to the CCN family, which sequesters VEGF in an angiogenesis-inhibiting complex. CTGF can be processed by MMPs and by ADAM28 which cleaves CTGF from the CTGF/VEGF 165 complex, releasing biologically active VEGF (Vascular Endothelial Growth Factor) that activates the VEGF/VEGFR2 signaling pathway [12] (Fig. 2). Immunohistochemistry analyses indicate a co-expression of ADAM28, CTGF and VEGF in breast carcinoma tissues supporting the hypothesis that ADAM28 stimulates VEGF-induced angiogenesis through the digestion of CTGF from the CTGF/VEGF complex. Interestingly, ADAM28 also cleaves other CCN family members i.e. Cyr61/CCN1, NOV/CCN3, WISP-1/CCN4, WISP-2/CCN5 and WISP-3/CCN6 but no further investigation has been pursued to determine the biological relevance of their cleavage by ADAM28.

\subsection{Epithelial-to-mesenchymal transition (EMT)}

EMT is a process characterized by phenotypic changes (e.g. loss of cell polarity and cell-cell adhesion) and the transcription factor SOX4 (SRY-related HMG-box 4) is now reported as one of the master regulators of EMT [73]. Co-transfection of COS-7 cells with ADAM28 promoter construct and SOX4 expression vector indicates that SOX4 activates ADAM28 reporter gene expression via its interaction with ADAM28 promoter [74]. Overexpression of SOX4 in lung and breast carcinoma cells enhances ADAM28 expression resulting in increased tumor cells migration. Moreover, co-localization of SOX4 and ADAM28 has been observed at the invasive front of human breast and lung carcinoma tissues. Altogether, these results demonstrate that ADAM28, which is transactivated by SOX4, promotes cell invasion of breast and lung carcinoma cells [74]. One can hypothesize that ADAM28 participates in SOX4-mediated EMT at invasive sites of tumors but the link remains to be clearly established (Fig. 2).

\subsection{Extracellular matrix degradation}

A key step in cancer progression is the detachment of cancer cells from the tumor followed by the invasion of the surrounding tissues. Hence, cancer cells then migrate through extracellular spaces by degrading components of the extracellular matrix and further intravasate into the bloodstream. Extracellular matrix (ECM) is a highly organized structural network composed of proteoglycans, collagens, elastin, fibronectin and laminin and these components are digested by proteases from the SVMPs and MMPs family which share a high homology sequence with ADAM28 that is also thought to be involved in 
ECM remodeling and thus, in tumor cell invasion [11,44] (Fig. 2). Both ADAM28m and ADAM28s isoforms exhibit proteoglycan degradation activities in vitro indicating that ADAM28 could be a new aggrecanase [20]. On the other hand, murine ADAM28 is not able to process matrix macromolecules such as collagen (types I, II, IV and V), laminin or fibronectin [11]. So far, no other components of the ECM have been identified as ADAM28 substrates.

\subsection{Cancer cell survival in bloodstream}

As tumor cells enter into the blood circulation where their survival is critical since they are more directly exposed to immune cells, they might elaborate some tricks that avoid their destruction by the immune system and favor their dissemination throughout the body. In this matter, ADAM28 proteolytic activities protect tumor cells entering the bloodstream from apoptosis triggered by von Willebrand Factor (vWF), a multifunctional glycoprotein produced by endothelial cells and megakaryocytes that displays complex roles in metastatic dissemination processes [14,75]. In the blood vasculature, plasma vWF circulates as a large multimeric complex which mainly originates from endothelial cells and is notably, implicated in tumor cell death. While cultured carcinoma cells expressing ADAM28 are resistant to vWF-induced apoptosis, blocking ADAM28 using a pharmacological inhibitor or an interference tool restores the apoptosis capacity of vWF. In vivo, mice intravenously injected with lung adenocarcinoma cells, whose ADAM28 expression is downregulated, display reduced lung metastasis and increased tumor cell apoptosis rates [52]. Therefore, one could hypothesize that the tumor-promoting effects of ADAM28 rely on an enhanced survival of carcinoma cells by protecting them from vWF-mediated cell death (Fig. 2).

\subsection{Formation of distant metastasis}

In NSCLC, ADAM28 expression is associated with lymph node metastasis but its implication in metastatic dissemination remains unknown. One hypothesis is that ADAM28 expressed by tumor cells might boost their adhesion and migration across the endothelium via the binding of the ADAM28 disintegrin domain to adhesion molecules present on vascular walls (Fig. 2). ADAM28 could increase migratory properties of melanoma cells expressing integrin $\alpha_{4} \beta_{1}$ by interacting with VCAM-1 expressed by activated endothelial cells [76]. In addition, ADAM28 may reinforce the interaction between PSGL-1/P-selectin by binding to PSGL-1, expressed by prostate tumor cells resulting in enhanced metastatic formation [77].

\section{Anti-cancer effects of ADAM28}

Although contribution of matrix metalloproteinases to cancer progression and dissemination is largely documented in the literature, tumor suppressive functions have also been ascribed to some of these proteases $[6,78,79]$. Recent studies have aroused dual functions for some members of ADAMTS and ADAM families meaning that a unique protease can exert both pro- and anti-tumor effects depending on its committed structural domain and expression pattern.

In colorectal cancer patients, ADAM28 has surprisingly been preferentially detected in adjacent non-tumor tissues [80]. Moreover, ADAM28 downregulation is associated with increased in vitro proliferation, migration and clonogenicity capacities of colorectal cancer cells suggesting that ADAM28 might play a tumor suppressor role in this cancer. In contrast, the miRNA-552 is found elevated in tumor tissues and colorectal cancer cell lines. As previous data indicate that ADAMs could be regulated by miRNAs in cancer, researchers investigate whether ADAM28 is a potential target of miRNA-552 and they actually validate the targeting of ADAM28 by miR-552. Therefore, we could speculate that pro-tumor effects of miRNA-552 are partially attributed to the direct targeting of ADAM28 resulting in its knockdown in colorectal cancer. Moreover, a recent study has revealed that colorectal cancer cells treated with miR-198 targeting ADAM28 expression display reduced proliferation and colony formation capacities but increased apoptosis rates [81]. Expression of ADAM28 in colorectal cancer cell lines (SW480 and SW620) has been found to reverse the effects induced by miR-198 and thus, to restore pro-tumor functions in cells. Implication of ADAM28 in colorectal cancer is not clearly understood since dysregulation of both miR-552 and miR-198 has been only evaluated in vitro on distinct cell lines. These studies indicate that ADAM28 exerts anti-tumor effects that may be strictly linked to the cancer type.

Recently, by using ADAM28 deficient mice, we reported that ADAM28-derived from microenvironment delays cancer cell dissemination suggesting a protective role against metastasis formation for ADAM28[33]. We observe that total depletion of ADAM28 enhances lung metastasis formation probably by reducing cytotoxic $\mathrm{T}$ cell infiltration of tumor-bearing lungs. As $\mathrm{CD}^{+} \mathrm{T}$ cells do not produce ADAM28, we hypothesize that ADAM28 confers protection against metastatic spread via indirect modulation of the cytotoxic immune response. Therefore, considering ADAM28 as potential therapeutic target is highly questionable.

\section{Conclusions and perspectives}

ADAM28 is upregulated in various types of tumor cells and its expression by cancer cells appears to act in favor of cancer development and progression with the exception of colorectal cancer. In sharp contrast, ADAM28 expressed by cells from the microenvironment displays protective effects against metastatic dissemination. This is a strong argument for not considering ADAM28 as a suitable therapeutic target.

Many research questions still require further investigations. All studies focusing on tumor cells overexpressing ADAM28 and published so far have been performed on human cell lines and therefore, requested to use immunodeficient mice with, as a consequence an oversight of the tumor-associated immune response. However, ADAM28 might impact tumor infiltration by immune cells and consequently modulate the immune response against cancer. Another challenge to understand the precise role of ADAM28 in cancer biology will be to assign novel specific substrates to ADAM28 since most of its known substrates are common with others ADAMs.

In conclusion, ADAM28 protease plays roles in different stages of cancer development and progression and could be used as a biomarker for different cancers. On the other hand, ADAM28 is also an anti-cancer molecule when produced by the stroma. These two faces make ADAM28 hazardous to consider as a therapeutic target.

\section{Author contributions}

Céline Hubeau wrote the manuscript, prepared figures and edited the manuscript. Natacha Rocks and Didier Cataldo critically revised the manuscript.

\section{Funding}

This study was financially supported by grants from the WB health program of the Walloon Region (WB Health AEROGAL, Convention no 1318023), the Fonds National pour la Recherche Scientifique (FRSFNRS Télévie, Grant no 7462914F, 7652716F and 7650218F), the Centre Anticancéreux (CAC, University of Liège), the Foundation against Cancer (foundation of public interest, Belgium), and the Foundation Léon Fredericq (University of Liège).

\section{Disclosure statement}

No disclosures to be made. 
Declaration of competing interestCOI

The authors have no conflicts of interest to disclose.

\section{References}

[1] P. Primakoff, H. Hyatt, J. Tredick-Kline, Identification and purification of a sperm surface protein with a potential role in sperm-egg membrane fusion, J. Cell Biol. 104 (1987) 141-149.

[2] T. Klein, R. Bischoff, Active metalloproteases of the A Disintegrin and Metalloprotease (ADAM) family: biological function and structure, J. Proteome Res. 10 (2011) 17-33.

[3] N. Rocks, et al., Emerging roles of ADAM and ADAMTS metalloproteinases in cancer, Biochimie 90 (2008) 369-379.

[4] M. Mullooly, P.M. McGowan, J. Crown, M.J. Duffy, The ADAMs family of proteases as targets for the treatment of cancer, Canc. Biol. Ther. 17 (2016) 870-880.

[5] L. Zadka, M.J. Kulus, K. Piatek, ADAM protein family - its role in tumorigenesis, mechanisms of chemoresistance and potential as diagnostic and prognostic factors, Neoplasma 65 (2018) 823-839.

[6] C. López-otín, L.M. Matrisian, Proteases have fundamental roles in multiple biological processes and are asso- ciated with a wide variety of pathological conditions, including cancer, Nature 7 (2007) 800-808.

[7] C.M. Roberts, P.H. Tani, L.C. Bridges, Z. Laszik, R.D. Bowditch, MDC-L, a novel metalloprotease disintegrin cysteine-rich protein family member expressed by human lymphocytes, J. Biol. Chem. 274 (1999) 29251-29259.

[8] L. Howard, R.A. Maciewicz, C.P. Blobel, Cloning and characterization of ADAM28: evidence for autocatalytic pro-domain removal and for cell surface localization of mature ADAM28, Biochem. J. 348 (2000) 21-27.

[9] S. Mochizuki, M. Shimoda, T. Shiomi, Y. Fujii, Y. Okada, ADAM28 is activated by MMP-7 (matrilysin-1) and cleaves insulin-like growth factor binding protein-3, Biochem. Biophys. Res. Commun. 315 (2004) 79-84.

[10] A.M. Fourie, F. Coles, V. Moreno, L. Karlsson, Catalytic activity of ADAM8, ADAM15, and MDC-L (ADAM28) on synthetic peptide substrates and in ectodomain cleavage of CD23, J. Biol. Chem. 278 (2003) 30469-30477.

[11] L. Howard, Y. Zheng, M. Horrocks, R.A. Maciewicz, C. Blobel, Catalytic activity of ADAM28, FEBS Lett. 498 (2001) 82-86.

[12] S. Mochizuki, et al., Connective tissue growth factor is a substrate of ADAM28, Biochem. Biophys. Res. Commun. 402 (2010) 651-657.

[13] Y. Mitsui, et al., ADAM28 is overexpressed in human breast carcinomas: implications for carcinoma cell proliferation through cleavage of insulin-like growth factor binding protein-3, Canc. Res. 66 (2006) 9913-9920.

[14] S. Mochizuki, et al., Effect of ADAM28 on carcinoma cell metastasis by cleavage of von willebrand factor, J. Natl. Cancer Inst. 104 (2012) 906-922.

[15] Y. Zhang, et al., CD19 regulates ADAM28-mediated Notch2 cleavage to control the differentiation of marginal zone precursors to MZ B cells, J. Cell Mol. Med. 21 (2017) 3658-3669.

[16] A.M. Babic, C.C. Chen, L.F. Lau, Fisp12/mouse connective tissue growth factor mediates endothelial cell adhesion and migration through integrin alphavbeta3, promotes endothelial cell survival, and induces angiogenesis in vivo, Mol. Cell Biol. 19 (1999) 2958-2966.

[17] T. Shimo, et al., Connective tissue growth factor induces the proliferation, migration, and tube formation of vascular endothelial cells in vitro, and angiogenesis in vivo, J. Biochem. 126 (1999) 137-145.

[18] C.-Y. Chu, C.-C. Chang, E. Prakash, M.-L. Kuo, Connective tissue growth factor (CTGF) and cancer progression, J. Biomed. Sci. 15 (2008) 675-685.

[19] I. Inoki, et al., Connective tissue growth factor binds vascular endothelial growth factor (VEGF) and inhibits VEGF-induced angiogenesis, Faseb. J. 16 (2002) 1-27.

[20] Y. Hikichi, K. Yoshimura, M. Takigawa, All-trans retinoic acid-induced ADAM28 degrades proteoglycans in human chondrocytes, Biochem. Biophys. Res. Commun. 386 (2009) 294-299.

[21] L.C. Bridges, et al., The lymphocyte metalloprotease MDC-L (ADAM 28) is a ligand for the integrin $\alpha 4 \beta 1$, J. Biol. Chem. 277 (2002) 3784-3792.

[22] L.C. Bridges, K.R. Hanson, P.H. Tani, T. Mather, R.D. Bowditch, Integrin $\alpha 4 \beta 1$ dependent adhesion to ADAM 28 (MDC-L) requires an extended surface of the disintegrin domain, Biochemistry 42 (2003) 3734-3741.

[23] O.J. McGinn, et al., Modulation of integrin $\alpha 4 \beta 1$ by ADAM 28 promotes lymphocyte adhesion and transendothelial migration, Cell Biol. Int. 35 (2011) 1043-1053.

[24] L.C. Bridges, D. Sheppard, R.D. Bowditch, ADAM disintegrin-like domain recognition by the lymphocyte integrins $\alpha 4 \beta 1$ and $\alpha 4 \beta 7$, Biochem. J. 387 (2005) $101-108$.

[25] M. Shimoda, et al., Binding of ADAM28 to P-selectin glycoprotein ligand-1 enhances P-selectin-mediated leukocyte adhesion to endothelial cells, J. Biol. Chem. 282 (2007) 25864-25874.

[26] Y. Miyamae, et al., ADAM28 is expressed by epithelial cells in human normal tissues and protects from C1q-induced cell death, FEBS J. 283 (2016) 1574-1594.

[27] A.L. Stone, M. Kroeger, Q.X.A. Sang, Structure-function analysis of the ADAM family of disintegrin-like and metalloproteinase-containing proteins (review), J. Protein Chem. 18 (1999) 447-465.

[28] D.F. Seals, S.A. Courtneidge, The ADAMs family of metalloproteases: multidomain proteins with multiple functions, Genes Dev. 17 (2003) 7-30.

[29] C. Rudnicka, et al., Overexpression and knock-down studies highlight that a disintegrin and metalloproteinase 28 controls proliferation and migration in human prostate cancer, Med. (United States) 95 (2016), 0-7.
[30] M. Asakura, et al., Cardiac hypertrophy is inhibited by antagonism of ADAM12 processing of HB-EGF: metalloproteinase inhibitors as a new therapy, Nat. Med. 8 (2002) 35-40.

[31] J. Oh, et al., Molecular, biochemical, and cellular characterization of epididymal ADAMs, ADAM7 and ADAM28, Biochem. Biophys. Res. Commun. 331 (2005) 1374-1383.

[32] I.D. Haidl, G. Huber, K. Eichmann, An ADAM family member with expression in thymic epithelial cells and related tissues, Gene 283 (2002) 163-170.

[33] C. Gérard, et al., Microenvironment-derived ADAM28 prevents cance dissemination, Oncotarget 9 (2018).

[34] K. Gossens, S. Naus, G.A. Holländer, H.J. Ziltener, Deficiency of the metalloproteinase-disintegrin ADAM8 is associated with thymic hyper-cellularity, PloS One 5 (2010) 1-9.

[35] L. Tian, et al., ADAM10 is essential for proteolytic activation of Notch during thymocyte development, Int. Immunol. 20 (2008) 1181-1187.

[36] J.O. Manilay, A.C. Anderson, C. Kang, E.A. Robey, Impairment of thymocyte development by dominant-negative kuzbanian (ADAM-10) is rescued by the Notch ligand, delta-1, J. Immunol. 174 (2005) 6732-6741.

[37] D.R. Gibb, S.J. Saleem, N.S. Chaimowitz, J. Mathews, D.H. Conrad, The emergence of ADAM10 as a regulator of lymphocyte development and autoimmunity, Mol. Immunol. 48 (2011) 1319-1327.

[38] N. Li, K. Boyd, P.J. Dempsey, D.A.A. Vignali, Non-cell autonomous expression of TNF- $\alpha$-converting enzyme ADAM17 is required for normal lymphocyte development, J. Immunol. 178 (2007) 4214-4221.

[39] N.A. Mabbott, D. Gray, Identification of co-expressed gene signatures in mouse B1, marginal zone and B2 B-cell populations, Immunology 141 (2014) 79-95.

[40] C.P. Blobel, et al., A potential fusion peptide and an integrin ligand domain in a protein active in sperm-egg fusion, Nature 356 (1992) 248-252.

[41] J.P. Evans, R.M. Schultz, G.S. Kopf, Mouse sperm-egg plasma membrane interactions: analysis of roles of egg integrins and the mouse sperm homologue of PH-30 (fertilin) beta, J. Cell Sci. 108 (Pt 1) (1995) 3267-3278.

[42] N. Giebeler, P. Zigrino, A disintegrin and metalloprotease (ADAM): historical overview of their functions, Toxins 8 (2016).

[43] C. Cho, The ADAM Family of Proteases, 2005

[44] J.A. Jury, Identification, sequence analysis and expression of transcripts encoding a putative metalloproteinase, eMDC II, in human and macaque epididymis, Mol. Hum. Reprod. 5 (1999) 1127-1134.

[45] Daiji Kiyozumi, et al., NELL2-mediated lumicrine signaling through OVCH2 is required for male fertility, Science 368 (6495) (2020) 1132-1135.

[46] H. Hammad, et al., Transitional B cells commit to marginal zone B cell fate by Taok3-mediated surface expression of ADAM10, Nat. Immunol. 18 (2017) 313-320.

[47] F. Martin, J.F. Kearney, Positive selection from newly formed to marginal zone B cells depends on the rate of clonal production, CD19, and btk, Immunity 12 (2000) 39-49.

[48] M. Acharya, et al., CD23/FceRII: molecular multi-tasking, Clin. Exp. Immunol. 162 (2010) 12-23.

[49] T. Twito, et al., Ectodomain shedding of CD200 from the B-CLL cell surface is regulated by ADAM28 expression, Leuk. Res. 37 (2013) 816-821.

[50] C. Ngwa, F. Liu, CD200-CD200R signaling and diseases: a potential therapeutic target? Int. J. Physiol. Pathophysiol. Pharmacol. 11 (2019) 297-309.

[51] J.R. Worley, D.A. Hughes, N. Dozio, J. Gavrilovic, M.J. Sampson, Low density lipoprotein from patients with Type 2 diabetes increases expression of monocyte matrix metalloproteinase and ADAM metalloproteinase genes, Cardiovasc. Diabetol. 6 (2007) 1-5.

[52] J.B.M. Jowett, et al., ADAM28 is elevated in humans with the metabolic syndrome and is a novel sheddase of human tumour necrosis factor- $\alpha$, Immunol. Cell Biol. 90 (2012) 966-973.

[53] J. Gacic, et al., Imatinib reduces cholesterol uptake and matrix metalloproteinase activity in human THP-1 macrophages, Pharmacol. Rep. 68 (2016) 1-6.

[54] L. Herat, et al., The metalloproteinase ADAM28 promotes metabolic dysfunction in mice, Int. J. Mol. Sci. 18 (2017).

[55] G. Gorfu, J. Rivera-Nieves, K. Ley, Role of beta 7 integrins in intestinal lymphocyte homing and retention, Curr. Mol. Med. 9 (2009) 836-850.

[56] S. Kon, T. Uede, The role of $\alpha 9 \beta 1$ integrin and its ligands in the development of autoimmune diseases, J. Cell Commun. Signal. 12 (2018) 333-342.

[57] N.M. Thielens, F. Tedesco, S.S. Bohlson, C. Gaboriaud, A.J. Tenner, C1q: A fresh look upon an old molecule, Mol. Immunol. 89 (2017) 73-83.

[58] A. Roemer, et al., Increased mRNA expression of ADAMs in renal cell carcinoma and their association with clinical outcome, Oncol. Rep. 11 (2004) 529-536.

[59] T. Ohtsuka, et al., ADAM28 is overexpressed in human non-small cell lung carcinomas and correlates with cell proliferation and lymph node metastasis, Int. J. Canc. 118 (2006) 263-273.

[60] H. Kuroda, et al., ADAM28 is a serological and histochemical marker for non-smallcell lung cancers, Int. J. Canc. 127 (2010) 1844-1856.

[61] X.H. Zhang, et al., ADAM28 overexpression regulated via the PI3K/Akt pathway is associated with relapse in de novo adult B-cell acute lymphoblastic leukemia, Leuk. Res. 39 (2015) 1229-1238.

[62] S. Matsuura, et al., Overexpression of A disintegrin and metalloproteinase 28 is correlated with high histologic grade in conventional chondrosarcoma, Hum. Pathol. 41 (2010) 343-351.

[63] M.H. Yang, et al., Characterization of ADAM28 as a biomarker of bladder transitional cell carcinomas by urinary proteome analysis, Biochem. Biophys. Res. Commun. 411 (2011) 714-720.

[64] L. Wei, et al., Oncogenic ADAM28 induces gemcitabine resistance and predicts a poor prognosis in pancreatic cancer, World J. Gastroenterol. 25 (2019) 5590-5603. 
[65] A. Stokes, et al., Expression profiles and clinical correlations of degradome components in the tumor microenvironment of head and neck squamous cell carcinoma, Clin. Canc. Res. 16 (2010) 2022-2035.

[66] H. Abe, et al., Src plays a key role in ADAM28 expression in v-src-transformed epithelial cells and human carcinoma cells, Am. J. Pathol. 183 (2013) 1667-1678.

[67] Y.L. Lv, et al., Baseline and decline of serum ADAM28 during chemotherapy of advanced non-small cell lung cancer: a probable predictive and prognostic factor, Med. Oncol. 29 (2012) 2633-2639.

[68] J.M. Zhang, et al., ADAM28 promotes tumor growth and dissemination of acute myeloid leukemia through IGFBP-3 degradation and IGF-I-induced cell proliferation, Canc. Lett. 442 (2019) 193-201.

[69] C.M. Wright, et al., ADAM28: a potential oncogene involved in asbestos-related lung adenocarcinomas, Gene Chromosome Canc. 49 (2010) 688-698.

[70] S. Mochizuki, Y. Okada, ADAMs in cancer cell proliferation and progression, Canc. Sci. 98 (2007) 621-628.

[71] S. Mochizuki, Y. Okada, ADAM28 as a target for human cancers, Curr. Pharmaceut. Des. 15 (2009) 2349-2358.

[72] E. Nowakowska-Zajdel, et al., Expression of ADAM28 and IGFBP-3 genes in patients with colorectal cancer - a preliminary report, Int. J. Immunopathol. Pharmacol. 26 (2013) 223-228.

[73] N. Tiwari, et al., Sox4 is a master regulator of epithelial-mesenchymal transition by controlling Ezh2 expression and epigenetic reprogramming, Canc. Cell 23 (2013) 768-783.
[74] A. Sasaki, H. Abe, S. Mochizuki, M. Shimoda, Y. Okada, SOX4, an epithelial-mesenchymal transition inducer, transactivates ADAM28 gene expression and co-localizes with ADAM28 at the invasive front of human breast and lung carcinomas, Pathol. Int. 68 (2018) 449-458.

[75] V. Terraube, I. Marx, C.V. Denis, Role of von Willebrand factor in tumor metastasis, Thromb. Res. 120 (Suppl) (2007) S64-S70.

[76] M. Klemke, T. Weschenfelder, M.H. Konstandin, Y. Samstag, High affinity interaction of integrin alpha4beta1 (VLA-4) and vascular cell adhesion molecule 1 (VCAM-1) enhances migration of human melanoma cells across activated endothelial cell layers, J. Cell. Physiol. 212 (2007) 368-374.

[77] C.J. Dimitroff, et al., Identification of leukocyte E-selectin ligands, P-selectin glycoprotein ligand-1 and E-selectin ligand-1, on human metastatic prostate tumor cells, Canc. Res. 65 (2005) 5750-5760.

[78] J. Decock, S. Thirkettle, L. Wagstaff, D.R. Edwards, Matrix metalloproteinases: protective roles in cancer, J. Cell Mol. Med. 15 (2011) 1254-1265.

[79] A. Noël, et al., New and paradoxical roles of matrix metalloproteinases in the tumor microenvironment, Front. Pharmacol. 3 (JUL) (2012) 1-9.

[80] J. Wang, et al., MicroRNA-552 enhances metastatic capacity of colorectal cancer cells by targeting a disintegrin and metalloprotease 28, Oncotarget 7 (2016) 70194-70210.

[81] L.X. Li, et al., MiR-198 affects the proliferation and apoptosis of colorectal cancer through regulation of ADAM28/JAK-STAT signaling pathway, Eur. Rev. Med. Pharmacol. Sci. 23 (2019) 1487-1493. 